\title{
PERFIL CLÍNICO DOS USUÁRIOS DA REDE DE ATENÇÃO PSICOSSOCIAL DE PICUÍ
}

\author{
CLINICAL PROFILE OF USERS OF THE PICUÍ PSYCHOSOCIAL \\ CARE NETWORK
}

\author{
Airy Ysmênia de Lima Medeiros ${ }^{1}$ \\ Cilânea dos Santos Costa ${ }^{2}$
}

RESUMO: Objetivo: O presente estudo teve como objetivo descrever o perfil clínico dos usuários da rede de saúde mental do município de Picuí - PB, além de fazer um diagnóstico das classes medicamentosas de psicotrópicos mais utilizadas. Método: Trata-se de um estudo de caráter quantitativo, com abordagem descritiva, considerando a prevalência dos psicotrópicos mais utilizados no município, bem como a predominância do gênero feminino e faixa etária descrita pelos Agentes Comunitários de Saúde. Para obtenção dos dados, foi realizado levantamento junto aos cadastros dos usuários do Município, no mês de agosto de 2019. Para compreensão da realidade estudada, usaram-se como parâmetros, informações referentes à faixa etária, sexo, dispensação de psicotrópicos e a média de consumo de medicamentos mensal. Resultados: Com a referida pesquisa, foi possível identificar lacunas que precisam ser melhoradas para a efetivação, ampliação e reformulação da RAPS no município, integrando todos os seus componentes de forma efetiva, no que se refere ao mapeamento e matriciamento em saúde mental, o fluxo de atendimento, projetos terapêuticos alternativos, busca ativa para cadastro de novos usuários, bem como resgatar os faltosos. Por fim, visando o acompanhamento do uso racional e seguro dos psicotrópicos e redução das prescrições excessivas pelas ESF, os resultados do estudo possibilitaram a implantação de ambulatório de saúde mental no município e a realização de capacitação com todos os profissionais da saúde. Conclusões: Conhecer o perfil do uso de psicotrópicos resultou na realização de um planejamento de acordo com as necessidades de saúde dos usuários e profissionais de saúde.

\footnotetext{
${ }^{1}$ Enfermeira Graduada pela Universidade Federal da Paraíba (UFPB). Especializanda em Vigilância em Saúde e em Avaliação de Saúde aplicada à Vigilância. Coordenadora de Vigilância em Saúde do município de Picuí-PB. Enfermeira assistencialista no Hospital de Trauma de Campina Grande. EMAIL: airysmenia@hotmail.com.

${ }^{2}$ Assistente Social graduada pela Universidade Estadual da Paraíba (UEPB). Especialista em Gestão Pública (UEPB) possui Residência Multiprofissional em Saúde Materno-Infantil da Universidade Federal do Rio Grande do Norte (UFRN). Atualmente é residente em Saúde Coletiva com Ênfase em Gestão de Redes de Saúde pela Escola de Governo em Saúde Pública de Pernambuco - ESPPE.
} 
Palavras chave: Atenção Primária em Saúde. Psicotrópicos. Saúde Mental.

\begin{abstract}
Objective: The present study aimed to describe the clinical profile of users of the mental health network in the city of Picui - PB, in addition to making a diagnosis of the most used psychotropic drug classes. Method: This is a quantitative study, with a descriptive approach, considering the prevalence of the most commonly used psychotropic drugs in the city, as well as the predominance of the female gender and age group described by the Community Health Agents. survey with the records of users of the Municipality, in the month of August 2019. To understand the reality studied, information related to the age group, sex, dispensation of psychotropics and the average monthly consumption of medicines were used as parameters. Results: With the aforementioned research, it was possible to identify gaps that need to be improved for the effectiveness, expansion and reformulation of RAPS in the municipality, effectively integrating all its components, with regard to mapping and matrixing in mental health, the flow service, alternative therapeutic projects, active search to register new users, as well as rescue the missing ones. Finally, aiming at monitoring the rational and safe use of psychotropic drugs and reducing excessive prescriptions by the FHS, the results of the study enabled the implantation of a mental health clinic in the city and the realization of training with all health professionals. Conclusions: Knowing the profile of the use of psychotropic drugs resulted in planning according to the health needs of users and health professionals.
\end{abstract}

Keywords: Primary Health Care. Psychotropic drugs. Mental Health. 


\section{INTRODUÇÃO}

O modelo de atendimento em saúde mental, no Brasil, vem sofrendo algumas alterações no decorrer desses últimos anos, desde a efetivação da reforma psiquiátrica. Dessa forma, a desinstitucionalização da assistência psiquiátrica, bem como o cuidado à saúde mental por meio de dispositivos extra-hospitalares e a inclusão da assistência dos usuários com transtorno na atenção básica são algumas das diretrizes da política de saúde mental do SUS. (Ministério da Saúde, 2009, p.348).

A Rede de Atenção Psicossocial (RAPS) foi criada para prestar uma melhor assistência às pessoas em sofrimento psíquico ou transtorno mental e com necessidades decorrentes do uso de crack, álcool e outras drogas no âmbito do Sistema Único de Saúde (SUS). Foi instituída pela Portaria № 3.088/2011, republicada em 21 de maio de 2013 e revogada pela Portaria de Consolidação $n^{\circ} 3$, de 28 de setembro de 2017, que trata da consolidação das normas sobre as redes do SUS. Foi disposta anteriormente pelo Decreto $N^{\circ} 7.508 / 2011$, o qual regulamente a Lei N 8.080 com objetivo de reorganizar o planejamento e assistência em saúde (BRASIL, 2017).

A RAPS está dividida em diversos componentes e pontos de atenção descritos a seguir: 
Quadro 1 - Componentes e Pontos de Atenção da RAPS.

\begin{tabular}{|c|c|}
\hline Componentes & Pontos de Atenção \\
\hline $\begin{array}{l}\text { Atenção Básica } \\
\text { em Saúde }\end{array}$ & $\begin{array}{l}\text {-Unidade Básica de Saúde (UBS); } \\
\text {-Equipes de Atenção Básica para populações em Situações } \\
\text { aEspecíficas (Consultório na Rua e Equipe de Apoio aos Serviços } \\
\text { do Componente Atenção Residencial de Caráter Transitório); } \\
\text { - Núcleo de Apoio à Saúde da Família (NASF); } \\
\text { - Centro de Convivência e Cultura }\end{array}$ \\
\hline $\begin{array}{l}\text { Atenção } \\
\text { Psicossocial }\end{array}$ & $\begin{array}{l}\text { Todas as modalidades de Centro de Atenção } \\
\text { Psicossocial (CAPS): CAPS I, CAPS II, CAPS III, CAPS AD } \\
\text { CAPS AD III, CAPS i }\end{array}$ \\
\hline $\begin{array}{l}\text { Atenção } \\
\text { Urgência } \\
\text { Emergência }\end{array}$ & $\begin{array}{l}\text { - Unidade de Pronto Atendimento (UPA); } \\
\text { e-Serviço de Atendimento Móvel de Urgência (SAMU); } \\
\text { - Sala de Estabilização; } \\
\text { - Portas Hospitalares de Atenção à Urgência/ Pronto-Socorro. }\end{array}$ \\
\hline $\begin{array}{l}\text { Atenção } \\
\text { Residencial } \\
\text { Caráter } \\
\text { Transitório }\end{array}$ & $\begin{array}{l}\text { e-Unidade de Acolhimento Adulto; } \\
\text { - Unidade de Acolhimento Infanto-Juvenil; } \\
\text {-Serviço de Atenção em Regime Residencial. }\end{array}$ \\
\hline \begin{tabular}{|l} 
Atenção \\
Hospitalar
\end{tabular} & $\begin{array}{l}\text {-Leitos de psiquiatria em Hospital Geral; } \\
\text { - Serviço Hospitalar de Referência. }\end{array}$ \\
\hline $\begin{array}{l}\text { Estratégias de } \\
\text { Desinstitucionali } \\
\text { zação }\end{array}$ & 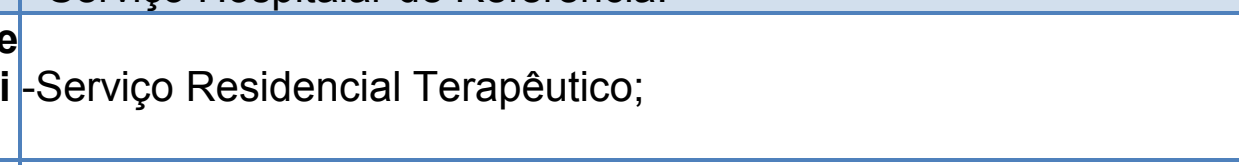 \\
\hline & -Programa de Volta para Casa. \\
\hline $\begin{array}{l}\text { Estratégias de } \\
\text { Reabilitação } \\
\text { Psicossocial }\end{array}$ & $\begin{array}{l}\text {-Cooperativas Sociais; } \\
\text {-Empreendimentos Solidários; } \\
\text { - Iniciativas de trabalho e Renda. }\end{array}$ \\
\hline
\end{tabular}

Fonte: (BRASIL, 2017)

Diante do exposto, podemos observar a importância da integração de todos os serviços citados como forma de ampliar e aprimorar a assistência prestada ao paciente com sofrimento psíquico em todo o seu contexto social.

Assim, é imprescindível entender que para se alcançar o aperfeiçoamento nos trabalhos voltados para a saúde mental, temos que comprovar que o manejo deste sofrimento ainda necessita de avanços na acessibilidade, na humanização e na criação de estratégias de promoção à saúde que abordem de forma globalizada as questões sociais e de relacionamento interpessoal que envolve os usuários desses serviços. 
Diante da magnitude e evolução dos transtornos mentais, torna-se relevante incentivar as investigações epidemiológicas na perspectiva de subsidiar informações concretas nessa área, bem como despertar em outros profissionais o interesse pela produção dos dados que caracterizem melhor a população que está sendo trabalhada, assim como a assistência que está sendo prestada, além de tentar ampliar e integrar todo o atendimento voltado aos pacientes de saúde mental do município de Picuí.

\section{METODOLOGIA}

Trata-se de um estudo de caráter quantitativo, com abordagem descritiva, considerando a prevalência dos psicotrópicos mais utilizados no município, bem como a predominância do gênero feminino e faixa etária descrita pelos Agentes Comunitários de Saúde.

Para obtenção dos dados, foi realizado durante todo o mês de agosto de 2019 um levantamento do quantitativo de usuários cadastrados pelos Agentes Comunitários de Saúde (ACS) do município que faziam uso de algum tipo de psicotrópico. Concomitantemente, foi realizado um levantamento na Farmácia Básica, através das fichas de distribuição de medicamentos, referente à dispensação de psicotrópicos para os usuários que procuram o referido serviço. Para compreensão da realidade estudada, usaram-se como parâmetros, informações referentes à faixa etária, sexo, dispensação de psicotrópicos e a média de consumo de medicamentos mensal.

Ao final, os dados foram analisados e contabilizados, servindo como parâmetro para a realização de pesquisas futuras, bem como para planejamento estratégico para aquisição de medicação psicotrópica anual e demais processos ou projetos que necessitem de dados relacionados aos supracitados. 


\section{RESULTADOS}

Embora o referido estudo não tenha tido a intenção de aprofundamento no conhecimento do perfil sociodemográfico dos usuários de psicotrópicos do munícipio, traçou- se de forma geral dados referentes à faixa etária e sexo para uma maior compreensão dos sujeitos que demandam os serviços de saúde mental. A tabela abaixo evidencia que o público feminino e em idade laborativa representa maior número de pessoas que fazem uso de psicotrópicos. Tal fato caminha em consonância com o contexto nacional, no qual os estudos apontam a existência de uma psiquiatrização das mulheres engendrada pelo modelo biomédico de fazer saúde, o qual converte os aspectos sociais e as subjetividades femininas como doença (CAMPOS, RAMALHO, ZANELO, 2017). Por outro lado, o mesmo estudo revelou que em se tratando do público masculino os conflitos familiares, relação conjugal, relação com os filhos e com mãe foram pouco evidenciados, uma vez que, as crises psíquicas aparecem estreitamente relacionadas aos sintomas descritos pela CID 10 (Classificação Internacional de Doenças).

Tabela 01 - Distribuição de usuários por faixa etária e gênero de acordo com as ESFs

\begin{tabular}{|c|c|c|c|c|c|c|c|c|c|}
\hline \multirow{2}{*}{ ESF } & \multicolumn{2}{|c|}{1 a 10 anos } & \multicolumn{2}{|c|}{11 a 30 anos } & \multicolumn{2}{|c|}{31 a 59 anos } & \multicolumn{2}{|c|}{$\begin{array}{c}60 \text { anos ou } \\
\text { mais }\end{array}$} & \multirow[t]{2}{*}{ Total } \\
\hline & M & $\mathbf{F}$ & M & $\mathbf{F}$ & $\mathbf{M}$ & $\mathbf{F}$ & M & $\mathbf{F}$ & \\
\hline 1 & 1 & - & 7 & 5 & 7 & 20 & 2 & 16 & 58 \\
\hline II & 9 & 7 & 24 & 37 & 44 & 114 & 14 & 50 & 285 \\
\hline III & 2 & 1 & 3 & 6 & 9 & 18 & 9 & 9 & 57 \\
\hline IV & 3 & - & 7 & 16 & 17 & 47 & 10 & 18 & 118 \\
\hline V & 7 & 3 & 22 & 20 & 36 & 73 & 23 & 87 & 271 \\
\hline VI & 1 & 1 & 1 & 2 & 4 & 19 & 5 & 5 & 38 \\
\hline VII & 1 & - & 3 & 5 & 2 & 13 & 4 & 12 & 39 \\
\hline VIII & 2 & - & 7 & 15 & 23 & 40 & 11 & 37 & 135 \\
\hline TOTAL & 26 & 12 & 74 & 106 & 142 & 344 & 78 & 234 & 1001 \\
\hline
\end{tabular}

Fonte: cadastro dos ACS. 
No que concerne ao uso dos psicotrópicos o estudo mostrou conforme pode ser visualizado na tabela a seguir que os benzodiazepínicos, antipsicóticos e antidepressivos estão entre os mais consumidos pela população picuiense. $\mathrm{O}$ uso indiscriminado dos benzodiazepínicos tem se tornado um caso de saúde pública, pois corresponde ao fenômeno de medicalização da vida. Os dados municipais também retratam a realidade nacional, uma vez que, as prescrições de tal psicotrópico têm sido efetuadas via atenção primária em saúde como estratégias terapeutas para tratamento de ansiedade e insônia, principais motivo do consumo (FEGADOLLI; VARELA; CARLINI, 2019).

Tabela 02 - Média de consumo de medicamentos mensal por classe e ESF.

\begin{tabular}{l|c|c|c|c|c}
\hline Medicação & Benzodiazepínico & Antipsicótico & Anticonvulsivante & Antidepressivo & Outro \\
\hline ESF I & 810 & 660 & 270 & 660 & 420 \\
\hline ESF II & 3.510 & 2.250 & 3.010 & 3.310 & 1.910 \\
\hline ESF III & 660 & 570 & 540 & 540 & 630 \\
\hline ESF IV & 1.080 & 1.320 & 810 & 960 & 1.230 \\
\hline ESF V & 3.780 & 2.790 & 1.860 & 2.460 & 1.560 \\
\hline ESF VI & 350 & 360 & 390 & 540 & 300 \\
\hline ESF VII & 390 & 630 & 330 & 510 & 330 \\
\hline ESF VIII & 2.370 & 2.250 & 1.590 & 1.680 & 1.500 \\
\hline Total/mês & $\mathbf{1 2 . 9 5 0}$ & $\mathbf{1 0 . 8 3 0}$ & $\mathbf{8 . 8 0 0}$ & $\mathbf{1 0 . 6 6 0}$ & $\mathbf{7 . 8 8 0}$ \\
\hline Total/ano & $\mathbf{1 5 5 . 4 0 0}$ & $\mathbf{1 2 9 . 9 6 0}$ & $\mathbf{1 0 5 . 6 0 0}$ & $\mathbf{1 2 7 . 9 2 0}$ & $\mathbf{9 4 . 5 6 0}$ \\
\hline
\end{tabular}

Fonte: fichas de distribuição de psicotrópicos.

Com base ainda no levantamento de dados, o referido estudo preocupou-se em fazer um comparativo entre os anos de 2011 e 2019, no tocante ao aumento do uso de psicotrópicos por Estratégia de Saúde da Família, sendo evidenciado que em 8 anos, houve um aumento de $155 \%$ no total de usuários. Entretanto, a ESF $V$ teve um aumento de $191 \%$, mesmo com a saída de pacientes para a ESF VIII. É oportuno destacar que no ano de 2011, a ESF VIII ainda não estava funcionando. Portanto, os pacientes estavam distribuídos nas ESFs IV e V. 
Gráfico 01 - comparativo de usuários que fazem uso de psicotrópico por ESF nos anos de 2011 e 2019.

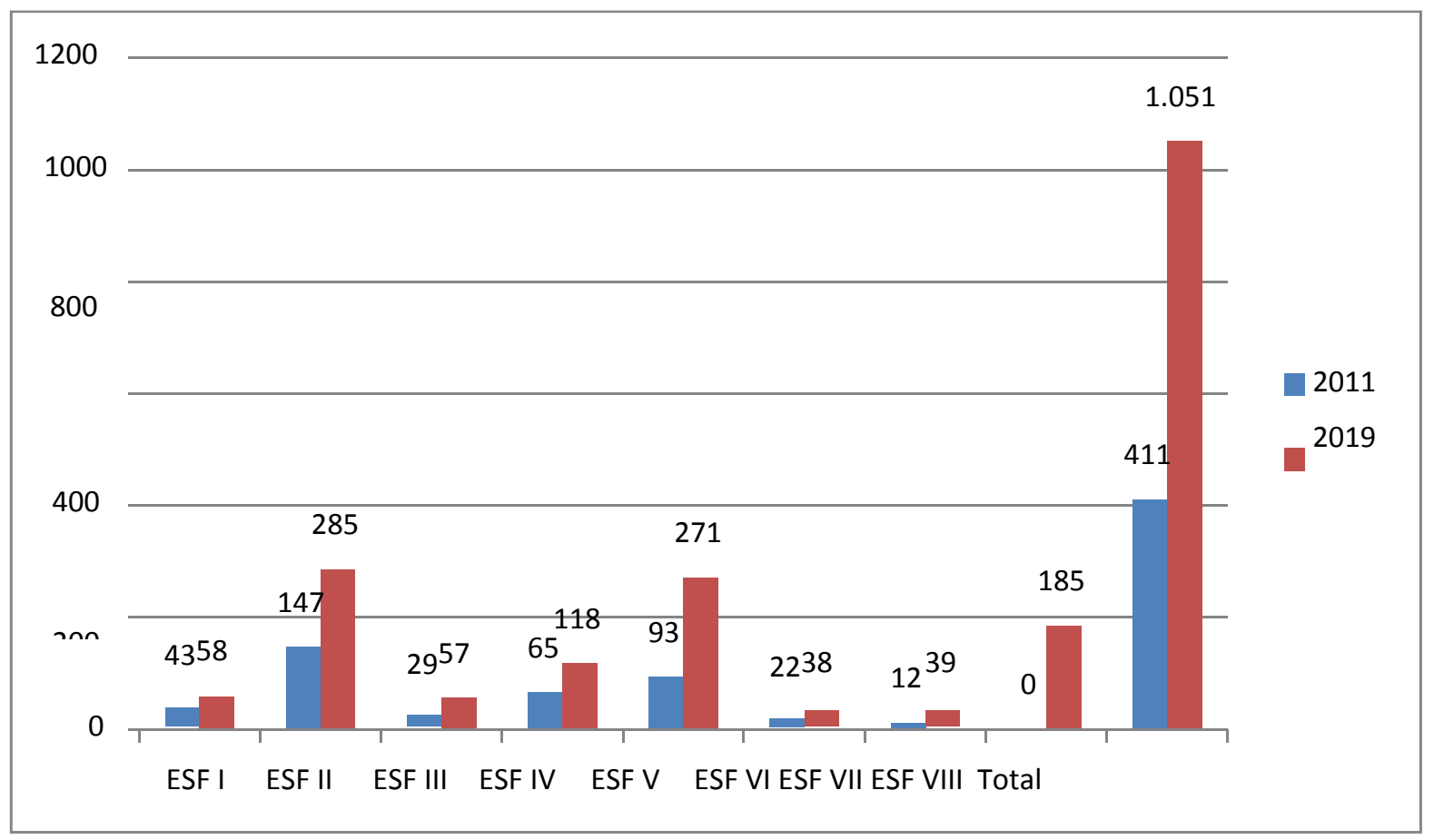

Fonte: Cadastro dos ACS

Os dados desse estudo reforçam que embora todo o esforço engendrado pela Reforma Psiquiátrica com seu modelo de pensar a saúde mental no país, ainda persistem traços das práticas biomédicas. A lógica da saúde mental na atenção básica é atravessada por fatores que se correlacionam entre si e suprimem o atendimento integral, a saber: fragilidade na formação e capacitação profissional; modelo biomédico medicalizante e excludente; falhas no encaminhamento e fragmentação do cuidado por especialidade; ausência de rede de apoio à família; ausência de práticas na comunidade que potencializem o cuidado da atenção básica e psicossocial no território (CAMPOS; BEZERRA; JORGE, 2020, p.4 ).

Diante do exposto, é cada vez mais urgente o envolvimento de todos os atores sejam gestores, usuários, profissionais e sociedade para que se possa garantir a inclusão social, cidadania e autonomia dos sujeitos. Sendo assim, as estratégias para transpor as barreiras perpassam pela educação permanente, 
reorganização do trabalho e dos serviços, políticas de implementação de recursos terapêuticos alternativos (FEGADOLLI; VARELA; CARLINI, 2019).

Destacamos que do total de usuários que receberam medicamento pela farmácia do município, pelo menos uma vez, de janeiro a agosto de 2019: 762 usuários, sendo 478 mulheres e 284 homens, prevalecendo o público feminino o maior demandante de psicotrópicos no município.

Ainda em sintonia com os dados da tabela acima podemos verificar que o cuidado ofertado pela Atenção Básica no campo da saúde mental tem se restringido a consulta médica e prescrição de medicamentos. Cabe ressaltar que, conforme relatório ambulatório (2019) o total de usuários cadastrados e acompanhados pelo CAPS contabilizava 62 pessoas (TABNET).

Diante desse cenário, vários atores asseveram a importância de privilegiar 0 espaço da Atenção Primária em Saúde, pois a partir dela é possível identificar as demandas reprimidas no território, uma vez que é nele que os sujeitos desenvolvem suas subjetividades e sociabilidades. Pontuam ainda, que na APS é possível realizar um acolhimento qualificado, visitas domiciliares e formação de grupos terapêuticos que favorecem a ampliação do cuidado integral e efetivo acerca do sofrimento psíquico (FERNANDES; MATSUKURA; LOURENÇO, 2018).

\section{CONSIDERAÇÕES FINAIS}

Ao avaliar o perfil clínico dos usuários de Saúde Mental em Picuí - PB, em consonância com os dados obtidos, constata-se que ainda há uma discrepância entre os cadastros dos ACS, da farmácia, bem como usuários atendidos pelo CAPS. embora saibamos que nem todos os usuários irão procurar a farmácia do município para aquisição dos psicotrópicos. Entretanto, é necessário um maior monitoramento, avaliação e acompanhamento de ambos os cadastros.

Observa-se que a integração da Rede de Atenção Psicossocial no município ainda precisa de ajustes para ser efetivada. É imprescindível que haja o fortalecimento dos serviços ofertados para que o usuário com algum tipo de 
transtorno possa ter um atendimento mais digno e coerente com o que preconiza as normas das novas políticas de saúde mental.

Sabe-se que o número de usuários não é fidedigna, já que algumas medicações não são distribuídas pelas Secretarias Municipal e Estadual de Saúde, o que leva o usuário a comprar sua medicação e não comparecer no setor de referência. Porém a diferença entre os dados obtidos pelos ACSs, pela farmácia e os encontrados no CAPS é gritante. Há lacunas que precisam ser melhor trabalhadas, a exemplo do trabalho integrado entre Atenção Básica e CAPS.

Outro ponto negativo é o preconceito e a falta de informação na sociedade, o que obriga alguns usuários a se esconderem em casa e negar o uso de psicotrópico, bem como a hipótese de alguma alteração mental.

O uso elevado de psicoterápicos, principalmente, dos benzodiazepínicos, mostra que esta é a prática contínua no atendimento dessa clientela. Também é comum encontrar receitas com um número elevado de medicamento para um único usuário, com o intuito do mesmo diminuir a frequência na ESF e CAPS. É salutar efetivar estratégias que promovam a redução do quantitativo de medicação usada, sem que tal atitude prejudique o usuário.

Diante de tantos pontos expostos, é necessário que o município trabalhe de forma inter e multidisciplinar no que diz respeito à Saúde Mental, valorizando as ações desenvolvidas de forma integrada e em rede, bem como suas atividades terapêuticas grupais e individuais, envolvendo todas as esferas assistenciais para que haja uma inclusão social e melhor acompanhamento da saúde mental. 


\section{REFERÊNCIAS BIBLIOGRÁFICAS}

BRASIL. Ministério da Saúde, Conselho Nacional das Secretarias Municipais de Saúde. O SUS DE A à Z: garantindo saúde nos municípios. $3^{\text {a }}$ ed. Brasília: Editora do Ministério da Saúde, 2009.

BRASIL. Ministério da Saúde. Portaria de Consolidação $\mathbf{n}^{\circ}$ 3. Consolidação das normas sobre as redes do Sistema Único de Saúde. Brasília, DF, 2017. Disponível em:<http://bvsms.saude.gov.br/bvs/saudelegis/gm/2017/prc0003_03_10_2017.htm>. Acesso em: 18 de agosto de 2019.

CAMPOS, Ioneide de Oliveira; RAMALHO, Walter Massa; ZANELLO, Valeska. Saúde mental e gênero: O perfil sociodemográfico de pacientes em um centro de atenção psicossocial. Estud. psicol. (Natal), Natal, v. 22, n. 1, p. 68-77, mar. 2017. Disponível em:<http://pepsic.bvsalud.org/scielo.php?script=sci_arttext\&pid=S1413294X2017000100008 \&lng=pt\&nrm=iso>. Acesso em: 10 fevereiro de 2020.

CAMPOS, Daniella Barbosa; BEZERRA, Indara Cavalcante; JORGE, Maria Salete Bessa. Produção do Cuidado em Saúde Mental: Práticas Territoriais Na Rede Psicossocial. Trab. educ. saúde, Rio de Janeiro, v. 18, n. 1, e0023167, 2020 . Disponível em $<$ http://www.scielo.br/scielo.php?script=sci_arttext\&pid=S1981-

$77462020000100502 \&$ Ing=pt\&nrm=iso>. Acesso: em 01 março de 2020.

FEGADOLLI, Claudia; VARELA, Niurka Maria Dupotey; CARLINI, Elisaldo Luis de Araújo. Uso e abuso de benzodiazepínicos na atenção primária à saúde: práticas profissionais no Brasil e em Cuba. Cad. Saúde Pública, Rio de Janeiro, v. 35, n. 6, e 00097718,2019. Disponível em $<$ http://www.scielo.br/scielo.php?script=sci_arttext\&pid=S0102-

311X2019000705007\&lng=pt\&nrm=iso>. acesso em: 29 fevereiro de 2020.

FERNANDES, Amanda Dourado Souza Akahosi; MATSUKURA, Thelma Simões; LOURENCO, Mariana Santos De Giorgio. Práticas de cuidado em saúde mental na Atenção Básica: identificando pesquisas no contexto brasileiro. Cad. Bras. Ter. Ocup., São Carlos, v. 26, n. 4 , p. 904-914, Dec. 2018 Disponivel em: $<$ http://www.scielo.br/scielo.php?script=sci_arttext\&pid=S2526-

$89102018000400904 \&$ Ing=en\&nrm=iso>. Acesso em: 01 março de 2020. 